\title{
Zika Virus Association with Microcephaly: The Power for Population Statistics to Identify Public Health Emergencies
}

\author{
Samuel J. Stratton, MD, MPH
}

Recent world attention has been drawn to a global Zika virus outbreak and an association in Brazil of microcephaly in newborn infants with the outbreak. The outbreak is being managed aggressively by local health authorities and world health organizations. Identification of the potential association of fetal exposure to Zika virus and risk of microcephaly has been a remarkable improvement in global health action when compared to the initial actions relative to the West Africa Ebola outbreak.

A Zika virus outbreak was reported in Brazil in early 2015. Separate from the Zika outbreak, in September 2015, Brazilian health authorities began to receive reports from local physicians of infants born with microcephaly. By October, the Brazilian health authorities identified an increase in birth prevalence of microcephaly in northeast Brazil, compared with previously reported estimates of approximately 0.5 cases of microcephaly /10,000 live births. By November 2015, the Brazil Ministry of Health reported an increase of microcephaly cases and possible association with Zika virus infection during pregnancy. At this point, the Pan American Health Organization (PAHO; Washington, DC USA) published an alert regarding the increase in occurrence of microcephaly in Brazil and possible association with Zika. Soon after the initial alert, PAHO released a December report that the identification of Zika virus RNA by reverse transcription-polymerase chain reaction (RT-PCR) in amniotic fluid samples from two pregnant women whose fetuses were found to have microcephaly by prenatal ultrasound, and the identification of Zika virus RNA from multiple body tissues (including the brain) of an infant with microcephaly who died in the immediate neonatal period. ${ }^{1}$ These events prompted world-wide alerts concerning the possible association of microcephaly with the outbreak of Zika virus infection. $^{2}$

The government of Brazil and the world health community are to be congratulated for exemplary work and ethics in the management of the Zika-microcephaly outbreak. Realize that Brazil is undergoing economic stress and, at the same time, preparing for upcoming summer Olympic Games. The political decision to alert the local community and world of the Zika-microcephaly outbreak undoubtable was very difficult for the leaders of Brazil; but, they made the correct and ethical decision. Additionally, the world health organizations, including $\mathrm{PAHO}$, were quick to listen to local health providers and alert the global community to the outbreak and the need to take preventive action to control the emergency.

An important aspect of the Zika event has been the use of simple population statistics in the initial identification and recognition of the emergency. As noted above, the measure of microcephaly prevalence within the affected population was an initial and convincing statistic to provide evidence for the need to quick action. For field surveillance, as well as ongoing research in public health emergencies, the use of prevalence and incidence are powerful tools for assessment and identification of health problems and challenges within an involved population.

Prevalence and incidence measures are measures of the frequency of an event or disease among a population. Prevalence is a measure of the proportion of a population affected by a condition of interest and commonly is measured in one of two ways:

Prevalence (Point) $=[\#$ of cases with condition/\# in specified population] at a point in time

Prevalence (Period) $=[\#$ cases with condition/\# in specified population] during a time period

It is important to realize that prevalence is a measure of the overall occurrence of a condition within a population and therefore measures cases that have occurred recently or in the past before a triggering event such as a disaster or disease outbreak. As opposed to the measure of incidence discussed below, prevalence measures new and already existing cases within a population.

Different from prevalence measures, incidence measures the change among non-affected members of the population to those that become affected. Incidence (cumulative) is a measure of new cases within the population during a specified time period:

Incidence (cumulative) $=[\#$ new cases/population at risk (not already a case)] during a time period

Incidence varies from prevalence measures by excluding existing cases and measuring new cases of a condition or disease during a time period. The incidence of new disease in a population can be measured more accurately using incidence rate, which corrects for cases as they occur in a population and for those leaving the population during the measured time period. ${ }^{3}$ While prevalence is a crude measure of disease burden within a population, incidence is a more robust measure of the development of new disease cases during a time period of interest. Both measures are potent tools in epidemiological analysis of public health emergencies and assessment of a population in disaster events.

Comparing prevalence or incidence measures of a condition within a population to established "baseline" or average rates of the condition is a tool that allows for identification of a problem within the population. This was the case for Zika in Brazil. With good local public health surveillance, reports of microcephaly were suspected to represent an increase in the condition. By using measures of prevalence when there was suspicion of increased cases and comparing that prevalence measure to the established baseline prevalence for microcephaly within the population, it was confirmed that a public health emergency existed. This allowed for mobilization of resources to address the emergency and 
subsequently to identify a Zika virus outbreak as strongly associated with the increase in microcephaly cases. Most public health professionals will agree that initial identification of health emergencies within a community is often delayed; yet, early identification allows for more effectively addressing a public health challenge. Additionally, ongoing monitoring of prevalence or incidence provides a measure of the effectiveness of actions taken to address an identified problem.

As described, baseline measures of prevalence and incidence are helpful for determining if a current measure represents a change in the health condition of a population. This type of data is maintained in databases sponsored by national health programs, as was the case in Brazil. World-wide baseline health data are maintained by the World Health Organization (WHO; Geneva, Switzerland) and are freely available in multiple languages through the organization web site. ${ }^{4}$

It is important to use standard epidemiologic format for evaluating population-based statistics. This is done by calculating the number of cases per 1,000 persons, 10,000 persons, or 100,000 persons depending upon the frequency of the measured condition. This allows for comparison of data across different databases and points in time. Researchers should also be aware that providing

\section{References}

1. Neurological syndrome, congenital malformations, and Zika virus infection. Implications for public health in the Americas-epidemiological alert. Washington, DC USA: World Health Organization, Pan American Health Organization Web site. http:// www.paho.org/hq/index.php?option $=$ com_docman\&task=doc_view\&Itemid $=270 \&$ gid=32405\&lang=en. Accessed February 21, 2016.

2. Schuler-Faccini L, Ribeiro EM, Feitosa IM, et al. Possible association between Zika virus infection and microcephaly - Brazil, 2015. MMWR Morb Mortal Wkly Rep. 2016;65(3):59-62. measures of precision for prevalence or incidence ratios is important for other researchers and users of population statistics. Preferred are standard 95\% confidence intervals which also provide a formal assessment of the margin of error for the data presented. Other potential causes for bias in data should also be recognized and reported because the intent of a population measure is to describe the health condition of an entire population of interest. Methods of assuring that measures of prevalence or incidence reflect the true statistic for an affected or study population are important. These methods may include randomized sampling of members of the population or forms of cluster analysis that can be managed to pre-determine that the whole population is represented in analysis.

In summary, the WHO and its members, including $\mathrm{PAHO}$ and the government of Brazil, are to be congratulated for identification of the Zika virus outbreak and its association with devastating cases of microcephaly. All have demonstrated best ethics and application of science in their approach to the health emergency. The event has also shown the power of measures of population disease frequency in early identification and monitoring of a public health emergency.

3. Measures of Disease Frequency. In: Aschengrau A, Seage GR, (eds). Essentials of Epidemiology in Public Health. 2nd ed. Sudbury, Massachusetts USA: Jones and Bartlett; 2008: 41-54.

4. WHO Country Profiles. World Health Organization Web site. www.who.int/countries/ en. Accessed February 21, 2016.

Online publication: March 4, 2016

doi:10.1017/S1049023X16000170 